\title{
Effects of Preoperative Drug Holiday On Sequester Separation and Treatment Outcome in Cancer Patients With Medication-Related Osteonecrosis of The Jaw
}

\author{
Mitsunobu Otsuru ( $\square$ ootsuru@nagasaki-u.ac.jp) \\ Nagasaki University Graduate School of Biomedical Sciences \\ Sakiko Soutome \\ Nagasaki University Graduate School of Biomedical Sciences \\ Kota Morishita \\ Nagasaki University Graduate School of Biomedical Sciences \\ Saki Hayashida \\ Nagasaki University Graduate School of Biomedical Sciences \\ Maho Murata \\ Nagasaki University Graduate School of Biomedical Sciences \\ Yukinari Takagi \\ Nagasaki University Graduate School of Biomedical Sciences \\ Miho Sasaki \\ Nagasaki University Graduate School of Biomedical Sciences \\ Misa Sumi \\ Nagasaki University Graduate School of Biomedical Sciences \\ Souichi Yanamoto \\ Nagasaki University Graduate School of Biomedical Sciences \\ Masahiro Umeda \\ Nagasaki University Graduate School of Biomedical Sciences
}

\section{Research Article}

Keywords: drug, sequester, cancer, osteonecrosis

Posted Date: October 13th, 2021

DOI: https://doi.org/10.21203/rs.3.rs-958449/v1

License: (c) (1) This work is licensed under a Creative Commons Attribution 4.0 International License. Read Full License 


\section{Abstract}

We aimed to investigate whether antiresorptive agent drug holidays contributed to the formation of sequester separation and treatment outcome in medication-related osteonecrosis of the jaw (MRONJ) patients whose primary disease was malignancy. A total of $105 \mathrm{MRONJ}$ patients with malignant tumors as the primary disease who underwent surgery at Nagasaki University Hospital and Kansai Medical University Hospital from January 2009 to December 2020 were included. We recorded patient age, sex, primary disease (solid cancer, non-solid cancer), MRONJ stage, type and administration period of antiresorptive agents, presence of diabetes, corticosteroid use, drug holiday period, white blood cell count, serum, albumin, serum creatinine, outcomes, and computed tomography findings. We examined the relationship between a drug holiday and sequester separation and outcome. Drug holiday of more than 90 days $(P=0.629)$ was not a significant factor for sequester separation in the univariate analysis. In the multivariate analysis, steroid use $(P=0.009)$ and serum albumin ( $P=0.003)$ were extracted as factors affecting prognosis; withdrawal for more than 90 days $(P=0.98)$ was not a significant factor. MRONJ patients with cancer as the primary disease should be operated early without drug holidays if their general condition is relatively good, clinical symptoms are strong, and quality of life is improved.

\section{Introduction}

Medication-related osteonecrosis of the jaw (MRONJ) is a known side effect of antiresorptive agents such as bisphosphonate (BP) and denosumab (Dmab) [1-2]. The American Association of Oral and Maxillofacial Surgeons (AAOMS) position paper on the treatment of MRONJ recommended a conservative therapy [2]. However, there are reports suggesting that surgical treatment is more effective than conservative therapy for MRONJ [3-5]. The authors also reported that surgical treatment was superior to conservative treatment in a retrospective multicenter study [6]. This raises a question whether a drug holiday for antiresorptive agents is necessary for surgical treatment. This is a particularly serious problem for MRONJ patients with malignant tumors as their primary disease. There must be a benefit of a drug holiday because even a short withdrawal may result in increased bone metastases and stronger pain. In addition, few patients are able to have a long-term drug holiday with antiresorptive agents. Therefore, we investigated whether an antiresorptive agent drug holiday contributed to the formation of sequester separation and treatment outcome in MRONJ patients whose primary disease was malignancy.

\section{Methods}

\section{Study design and patients}

This was a retrospective observational study. Of $175 \mathrm{MRONJ}$ patients with malignant tumors as the primary disease who underwent surgery at Nagasaki University Hospital and Kansai Medical University Hospital from January 2009 to December 2020, 70 who underwent only conservative therapy were excluded, and 105 who underwent surgery were included in this study.

\section{Variables}

We recorded the following patient data: age, sex, primary disease (solid cancer, non-solid cancer), MRONJ stage, type of antiresorptive agent, administration period of antiresorptive agent, presence of diabetes, corticosteroid use, drug holiday period, white blood cell count, serum, albumin, serum creatinine, outcomes, and computed tomography (CT) findings. The MRONJ stage was determined the time of initial diagnosis and according to the AAOMS (2014). CT findings were evaluated by a consensus of the oral surgeon and radiologist using preoperative images for the presence or absence of the extent of the osteolytic area (localized or extended) and periosteal reaction. Osteolysis was classified as localized (the maxilla does not extend to the floor of the nasal cavity or maxillary sinus floor, the mandible extends above the mandibular canal), extended (the maxilla extends to the floor of the nasal cavity or maxillary sinus floor, the mandible includes the lower mandibular canal). The sequester separation was defined as a clear and almost total separation between the sequestrum and the normal bone (Figure 1). The drug holiday period was defined as the period from the next scheduled dose of antiresorptive agent after the last dose to the date of preoperative CT scan for the diagnosis of sequester separation and from the date of surgery to the examination of the outcome. Postoperative follow-up was based on CT imaging every 3 months, and imaging was performed as needed when clinical symptoms were strong. The diagnosis of cure was made when there was no osteolysis progression on CT and all clinical symptoms, including bone exposure had disappeared. We examined the relationship between a drug holiday and sequester separation and outcome.

\section{Statistical analyses}

All statistical analyses were performed using SPSS software (version 26.0; Japan IBM Co., Ltd., Tokyo, Japan), and a two-tailed P-value $₫ 0.05$ was considered significant. The correlation between each variable and separation of sequester was analyzed using the Mann-Whitney $U$ nonparametric test for continuous variables and Fisher's exact test for categorical variables. The correlation between each variable and treatment outcome was analyzed using univariate and multivariate cox regression analyses. The relationship between a drug holiday and treatment outcome was illustrated using the Kaplan-Meier method.

\section{Ethical approval}

The study protocol conformed to the ethical guidelines of the Declaration of Helsinki and the Ethical Guidelines for Medical and Health Research involving Human Subjects by the Ministry of Health, Labor and Welfare of Japan. This study was approved by the Institutional Review Board (\#21021509) of Nagasaki University Hospital. The need of informed consent was waived by the IRB of Nagasaki University Hospital Because this was a retrospective study, the research plan was published on the homepage of the participating hospitals according to the instructions of the IRB in accordance with the guaranteed optout opportunity.

\section{Data availability}


The datasets used and analyzed during the study are available from the corresponding author upon reasonable request.

\section{Results}

\section{Patient characteristics}

The number of MRONJ patients whose primary disease was a malignancy and who underwent surgical treatment was 105 (Table 1). Nine patients (8.6\%) underwent sequester separation and 96 (91.4\%) did not. There were 17 patients who had drug holidays for antiresorptive agents for more than 90 days before preoperative CT, 86 who did not, and 2 patients with no information available.

\section{Factors related to separation of sequester}

A drug holiday of more than 90 days $(\mathrm{P}=0.629)$ was not a significant factor for sequester separation in the univariate analysis (Table 2). No other significant factors were found.

\section{Relationship between a drug holiday and treatment outcome}

Use of steroid $(\mathrm{P}=0.032)$ and serum albumin $(\mathrm{P}=0.020)$ were identified as factors associated with prognosis in univariate analysis. Use of steroid $(\mathrm{P}=0.009)$ and serum albumin $(P=0.003)$ were further identified as factors affecting prognosis in multivariate analysis. Withdrawal of medication for more than 90 days $(P=0.98)$ was not a significant factor (Table 3$)$. There was no significant difference in the healing rate depending on the drug holiday period among patients with and without drug withdrawal: 60 days or more $(P=0.344), 90$ days or more $(P=0.899)$, and 120 days or more $(P=0.556)(F i g u r e 2)$.

\section{Discussion}

Whether surgical or conservative treatment is the best strategy for MRONJ is a matter of debate. In a retrospective multicenter study, the authors reported the superiority of surgical therapy over conservative therapy [6]. Therefore, at the authors' institution, surgery is indicated for patients with MRONJ whose primary disease is stable, clinical symptoms are strong, and quality of life can be improved by surgery. MRONJ, whose primary disease is cancer, is no exception. Of course, there is a difference in backgrounds between patients with malignancy and osteoporosis as the primary disease of MRONJ. Patients with malignant tumors use higher doses of antiresorptive agents and are also more likely to have lowered immunocompetence due to anticancer drugs and poor general health. The malignancy may progress rapidly. Therefore, at the authors' institution, surgery is performed after close discussion of the primary disease with the primary physician. In addition, the authors discussed preoperative drug holidays with the primary care physician, and in principle, surgery is often performed without a drug holiday.

In 2009, the AAOMS position paper recommended a 3-month drug holiday for low-dose antiresorptive agents before invasive dental surgery. However, the recommendation was modified in 2011 to operate without a drug holiday even with low-dose antiresorptive agents [2]. However, some reports suggest that a drug holiday should be considered in patients who have received oral antiresorptive agents for less than 4 years, and others advocate a short drug holiday [2, 7, 8]. Therefore, the drug holiday for antiresorptive agents before surgery is controversial. However, these discussions refer to oral, low-dose antiresorptive agents. Antiresorptive agents used for malignant tumors are mainly administered intravenously in high doses. Hayashida et al. reported that preoperative drug holidays did not improve surgical outcomes in MRONJ patients using low- or high-dose antiresorptive agents, but there are few reports on drug holidays for malignancies [9].

In this study, the authors examined the benefits of preoperative drug holidays specifically in MRONJ patients with malignancy as the primary disease. Although the preoperative drug holiday depended on the progression of the cancer, we considered 2-3 months to be the maximum in consideration of the postoperative healing period. Since sequester separation by a drug holiday may facilitate surgical operation and reduce surgical invasion, we investigated whether sequester separation was accelerated by a drug holiday and whether a drug holiday improves the cure rate. In this study, only about $10 \%$ of patients had sequester separation, and a drug holiday of more than 3 months had no effect on sequester separation. A longer drug holiday may promote sequester separation. However, too long antiresorptive agent drug holidays are unacceptable for cancer patients. Although sequester separation may reduce surgical invasiveness, it was thought to be less effective after about 3 months and ultimately not beneficial for the patient.

In addition, surgery with a drug holiday for MRONJ has no effect on the outcomes. Instead, it was thought to be dependent on systemic conditions such as steroid use and low nutrition due to low albumin levels. Considering these factors, MRONJ patients with cancer as the primary disease should be operated early without a drug holiday if their general condition is relatively good, clinical symptoms are strong, and quality of life is improved.

There are some limitations in the study. First, this is a retrospective analysis using a small number of patients; therefore, it is unclear whether the results obtained can be generalized. Second, this study was a cross-sectional study and did not longitudinally observe the effects of a drug holiday. However, as far as we know, this is the first study to investigate how a preoperative drug holiday affects sequester separation and treatment outcome in patients receiving high-dose antiresorptive agent therapy. We would like to increase the number of cases in the future and conduct a more detailed examination to confirm these results.

\section{Declarations}

\section{Funding}

Not Applicable 


\section{Conflicts of interest/Competing interests}

Not Applicable

\section{Authors' contributions}

All authors contributed to the study conception and design. Material preparation, data collection, and analysis were performed by K.M, S.H, M.M, Y.T, M.Sasaki, M.Sumi, S.Y, M.U. The first draft of the manuscript was written by M.O and S.S and all authors commented on previous versions of the manuscript. All authors read and approved the final manuscript.

\section{References}

1. Yarom, N. et al. Medication-related osteonecrosis of the jaw: MASCC/ISOO/ASCO Clinical Practice Guideline. J. Clin. Oncol, 37, 2270-2290 (2019).

2. Ruggiero, S. L. et al. American Association of Oral and Maxillofacial Surgeons Position Paper on medication-related osteonecrosis of the jaw-2014 Update. J. Oral. Maxillofac. Surg, 72,, 1938-1956 (2014).

3. Rupel, K. et al. A systematic review of therapeutical approaches in bisphosphonates-related osteonecrosis of the jaw (BRONJ). Oral Oncol, 50, 1049-1057 (2014).

4. Fliefel, R., Tröltzsch, M., Kühnisch, J., Ehrenfeld, M. \& Otto, S. Treatment strategies and outcome of bisphosphonate-related osteonecrosis of the jaw (BRONJ) with characterization of patients: a systematic review. Int. J. Oral Maxillofac. Surg, 44, 568-585 (2015).

5. Khan, A. A. et al. Diagnosis and management of osteonecrosis of the jaw: a systematic review and international consensus. J. Bone Miner. Res, 30, 3-23 (2015).

6. Hayashida, S. et al. Evaluation of the treatment strategies for medication-related osteonecrosis of the jaws (MRONJ) and the factors affecting treatment outcome: A multicenter retrospective study with propensity score matching analysis. J. Bone Miner. Res, 32, 2022-2029 (2017).

7. United States Food and Drug Administration: Background docu-ment for meeting of advisory committee for reproductiveRUGGIERO ET AL1953 health drugs and drug safety and risk management advisorycommittee. September 9, 2011. Available at:

http://www.fda.gov/downloads/AdvisoryCommittees/CommitteesMeetingMaterials/drugs/DrugSafetyandRiskManagementAdvisoryCommittee/ucm270؛ Accessed August 31, 2021

8. Damm, D. D. \& Jones, D. M. Bisphosphonate-related osteonecrosis ofthe jaws: A potential alternative to drug holidays. Gen. Dent, 61, 33-38 (2013).

9. Hayashida, S. et al. Drug holiday clinical relevance verification for antiresorptive agents in medication-related osteonecrosis cases of the jaw. J. Bone Miner. Metab, 38, 126-134 (2020).

\section{Tables}


Table 1 Patient characteristics

\begin{tabular}{|c|c|c|}
\hline \multicolumn{2}{|l|}{ Variable } & \multirow{2}{*}{$\begin{array}{l}\text { number of patients / mean } \pm \text { SD } \\
45\end{array}$} \\
\hline Sex & $\operatorname{man}$ & \\
\hline & woman & 60 \\
\hline Age & (years) & $70.0 \pm 11.0$ \\
\hline \multirow[t]{2}{*}{ Primary disease } & solid cancer & 90 \\
\hline & non-solid cancer & 15 \\
\hline \multirow[t]{2}{*}{ MRONJ site } & upper jaw & 40 \\
\hline & lower jaw & 65 \\
\hline \multirow[t]{3}{*}{ MRONJ stage } & stage 1 & 11 \\
\hline & stage 2 & 70 \\
\hline & stage 3 & 24 \\
\hline \multirow[t]{3}{*}{ Antiresorptive agent } & BP & 38 \\
\hline & Dmab & 43 \\
\hline & $\mathrm{BP} \rightarrow \mathrm{Dmab}$ & 24 \\
\hline \multirow[t]{2}{*}{ Use of steroid } & $(-)$ & 83 \\
\hline & $(+)$ & 22 \\
\hline \multirow[t]{2}{*}{ Diabetes } & $(-)$ & 81 \\
\hline & $(+)$ & 24 \\
\hline \multirow[t]{3}{*}{ Duration of admministration } & $<4$ years & 68 \\
\hline & $\geqq 4$ years & 35 \\
\hline & unknown & 2 \\
\hline Duration of drug holiday before surgery & (days) & $54.9 \pm 113.9$ \\
\hline \multirow[t]{3}{*}{ Drug holiday before surgery $\geqq 90$ days } & $(-)$ & 86 \\
\hline & $(+)$ & 17 \\
\hline & unknown & 2 \\
\hline Duration of drug holiday before CT & (days) & $33.3 \pm 70.9$ \\
\hline \multirow[t]{3}{*}{ Drug holiday before $C T \geqq 90$ days } & $(-)$ & 86 \\
\hline & $(+)$ & 17 \\
\hline & unknown & 2 \\
\hline Leukocytes & $(/ \mu \mathrm{L})$ & $6326 \pm 2570$ \\
\hline Albumin & $(g / d L)$ & $3.70 \pm 0.513$ \\
\hline Creatinine & $(\mathrm{mg} / \mathrm{dL})$ & $0.885 \pm 0.351$ \\
\hline \multirow[t]{2}{*}{ Extent of osteolytic area } & localized & 77 \\
\hline & extended & 28 \\
\hline \multirow[t]{2}{*}{ Periosteal reaction } & $(-)$ & 77 \\
\hline & $(+)$ & 28 \\
\hline \multirow[t]{2}{*}{ Separation of sequester } & $(-)$ & 96 \\
\hline & $(+)$ & 9 \\
\hline Total & & 109 \\
\hline \multicolumn{3}{|l|}{ Abbreviation } \\
\hline MRONJ:medication-related osteonecrosis of the jaw & & \\
\hline
\end{tabular}


Table 2 Relationship between each variable and sequester separation (univariate analysis)

\begin{tabular}{|c|c|c|c|c|}
\hline Variable & & sequester separation $(-)$ & sequester separation (+) & $p$ value \\
\hline Sex & man & 42 & 3 & 0.546 \\
\hline & woman & 54 & 6 & \\
\hline Age & (years) & $69.6 \pm 10.8$ & $70.9 \pm 12.2$ & 0.731 \\
\hline Primary disease & solid cancer & 83 & 7 & 0.477 \\
\hline & non-solid cancer & 13 & 2 & \\
\hline MRONJ site & upper jaw & 37 & 3 & 0.758 \\
\hline & lower jaw & 59 & 6 & \\
\hline MRONJ stage & stage 1 & 9 & 2 & 0.389 \\
\hline & stage 2 & 64 & 6 & \\
\hline & stage 3 & 23 & 1 & \\
\hline Antiresorptive agent & BP & 35 & 3 & 0.728 \\
\hline & Dmab & 40 & 3 & \\
\hline & $\mathrm{BP} \rightarrow \mathrm{Dmab}$ & 21 & 3 & \\
\hline Use of steroid & $(-)$ & 74 & 9 & 0.106 \\
\hline & $(+)$ & 22 & 0 & \\
\hline Diabetes & $(-)$ & 73 & 8 & 0.380 \\
\hline & $(+)$ & 23 & 1 & \\
\hline Duration of admministration & $<4$ years & 62 & 6 & 0.908 \\
\hline & $\geqq 4$ years & 32 & 3 & \\
\hline & unknown & 2 & 0 & \\
\hline Drug holiday $\geqq 90$ days & $(-)$ & 79 & 7 & 0.629 \\
\hline & $(+)$ & 15 & 2 & \\
\hline Leukocytes & $(/ \mu \mathrm{L})$ & $6371 \pm 2626$ & $5800 \pm 1830$ & 0.549 \\
\hline Albumin & $(g / d L)$ & $3.69 \pm 0.520$ & $3.80 \pm 0.432$ & 0.576 \\
\hline Creatinine & $(\mathrm{mg} / \mathrm{dL})$ & $0.893 \pm 0.356$ & $0.789 \pm 0.290$ & 0.424 \\
\hline Extent of osteolytic area & localized & 72 & 5 & 0.207 \\
\hline & extended & 24 & 4 & \\
\hline Periosteal reaction & $(-)$ & 72 & 5 & 0.207 \\
\hline & $(+)$ & 24 & 4 & \\
\hline Total & & 97 & 12 & \\
\hline Abbreviation & & & & \\
\hline MRONJ:medication-related osteonecr & is of the jaw & & & \\
\hline BP: bisphosphonate & & & & \\
\hline
\end{tabular}


Table 3 Relationship between each variable and treatment outcome (univariate and multivariate cox regression)

\begin{tabular}{|c|c|c|c|c|c|c|c|}
\hline \multirow[t]{2}{*}{ Variable } & & \multicolumn{3}{|c|}{ univariate analysis } & \multicolumn{3}{|c|}{ multivariated analysis } \\
\hline & & $p$ value & HR & $95 \% \mathrm{Cl}$ & $p$ value & HR & $95 \% \mathrm{Cl}$ \\
\hline Sex & man / woman & 0.363 & 1.398 & $0.679-2.880$ & & & \\
\hline Age & (years) & 0.653 & 1.008 & $0.975-1.042$ & & & \\
\hline Primary disease & solid cancer / non-solid cancer & 0.451 & 1.589 & $0.478-5.259$ & & & \\
\hline MRONJ site & upper jaw / lower jaw & 0.349 & 0.693 & $0.322-1.491$ & & & \\
\hline MRONJ stage & stage 1 / 2 / 3 & 0.725 & 1.128 & $0.579-2.200$ & & & \\
\hline Antiresorptive agent & $\mathrm{BP} / \mathrm{Dmab} / \mathrm{BP} \rightarrow \mathrm{Dmab}$ & 0.077 & 1.547 & $0.954-2.508$ & & & \\
\hline Use of steroid & $(-) /(+)$ & *0.032 & 0.374 & $0.152-0.918$ & *0.009 & 0.252 & $0.089-0.710$ \\
\hline Diabetes & $(-) /(+)$ & 0.134 & 0.448 & 0.157-1.279 & & & \\
\hline Duration of admministration & $<4$ years $/ \geqq 4$ years & 0.916 & 0.961 & $0.460-2.010$ & & & \\
\hline Drug holiday before surgery $\geqq 90$ days & $(-) /(+)$ & 0.954 & 1.037 & $0.308-3.491$ & 0.98 & 1.012 & $0.388-2.643$ \\
\hline Leukocytes & $(/ \mu \mathrm{L})$ & 0.988 & 1.000 & $1.000-1.000$ & & & \\
\hline Albumin & $(g / d L)$ & *0.020 & 0.423 & $0.204-0.875$ & *0.003 & 0.327 & $0.157-0.682$ \\
\hline Creatinine & $(\mathrm{mg} / \mathrm{dL})$ & 0.988 & 1.011 & $0.241-4.237$ & & & \\
\hline Extent of osteolytic area & localized / extended & 0.198 & 1.715 & 0.755-3.894 & & & \\
\hline Periosteal reaction & $(-) /(+)$ & 0.579 & 1.228 & $0.594-2.538$ & & & \\
\hline Separation of sequester & $(-) /(+)$ & 0.221 & 2.129 & $0.635-7.140$ & & & \\
\hline \multicolumn{8}{|l|}{ *significant } \\
\hline \multicolumn{8}{|l|}{ Abbreviation } \\
\hline \multicolumn{8}{|l|}{ MRONJ:medication-related osteonecrosis of the jaw } \\
\hline \multicolumn{8}{|l|}{ BP: bisphosphonate } \\
\hline Dmab: denosumab & & & & & & & \\
\hline
\end{tabular}

\section{Figures}




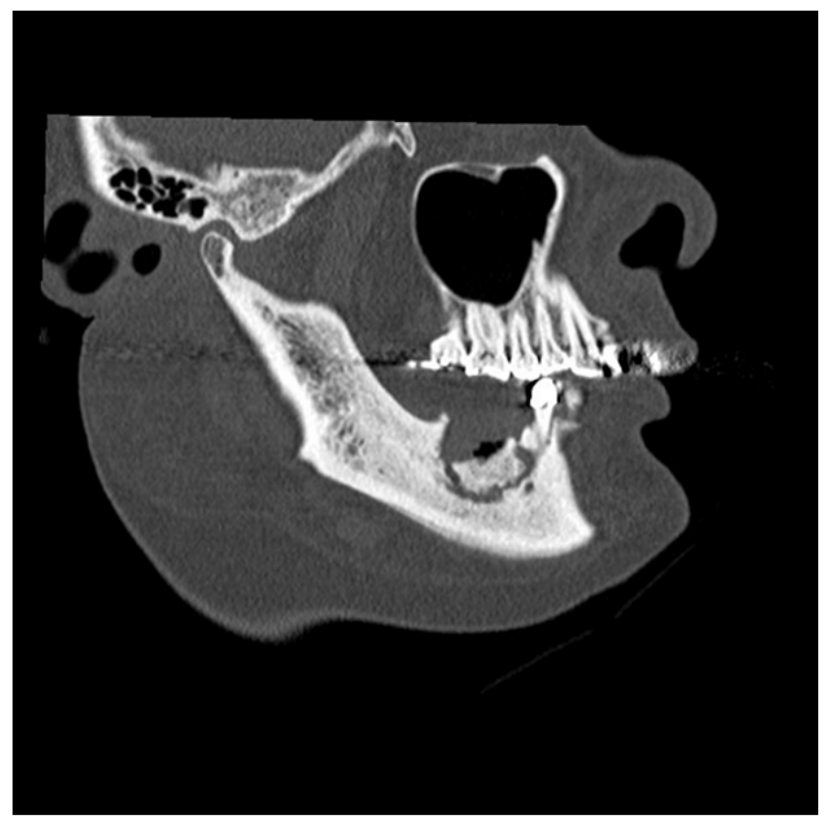

Figure 1

Sequester separation (computed tomography sagittal image): The sequester separation was defined as a clear and almost total separation between the sequestrum and the normal bone. 
a

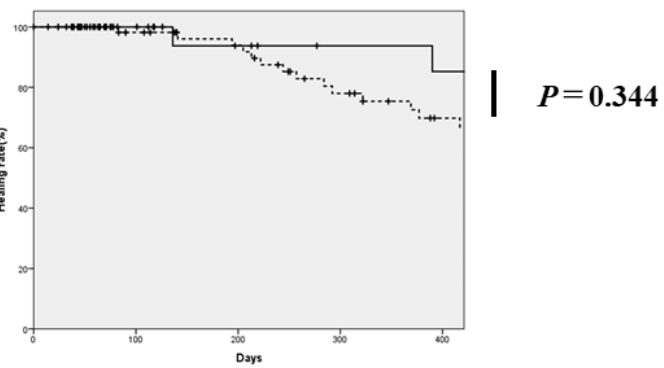

b

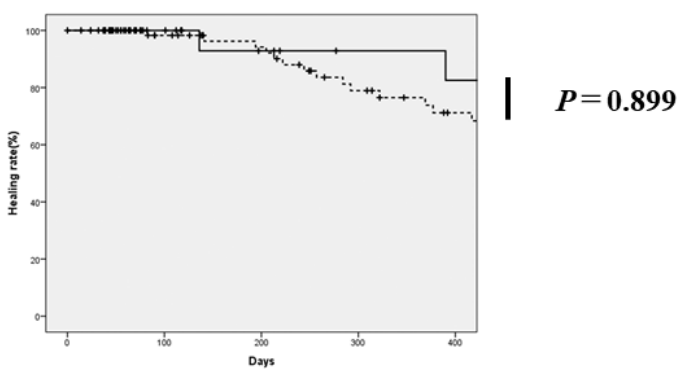

c

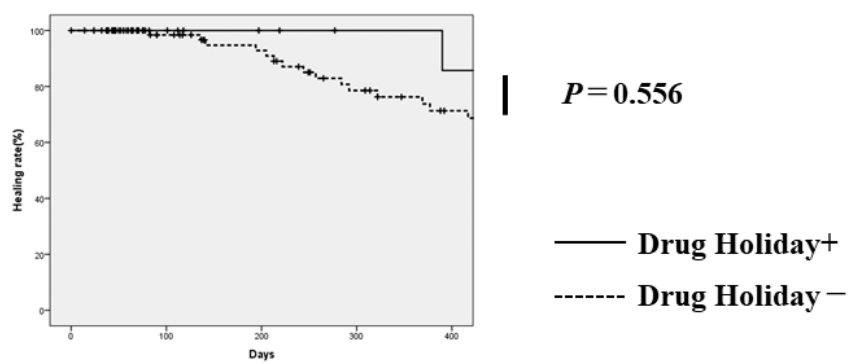

Figure 2

Healing rate depending on whether there is a drug holiday or not and the period of the drug holiday. a. Drug holiday for 60 days b. Drug holiday for 90 days $\mathrm{c}$. Drug holiday for 120 days 